FVC $>10 \%$ from baseline to 2 nd visit. For predicting models, predictors with $p<0.2$ in the univariate analysis were included in the logistic regression analysis.

Results: 8013 LcSSc were included with a mean follow-up of about $3.3 \pm 3.7$ years. At baseline, mean \pm SD mRSS was $6 \pm 5$ and ILD was present in $28.4 \%$ of all patients.

Worsening of skin fibrosis was observed in 6.4\% (19/298), 7.8\% (97/1248) and $9.8 \%(289 / 2957)$ of LcSSc patients at 6,12 and 24 months follow-up respectively. In multivariate analysis, variables predicting skin fibrosis progression were elevated European Scleroderma Study Group activity index (EScSG-AI) (OR [95 IC]: 1.22 [1.05-1.4], $p=0.007)$ for 12 months progression and EScSG-Al (1.24 [1.131.38], $\mathrm{p}<0.001)$ and mRSS (0.95 [0.93-0.98], $\mathrm{p}=0.001)$ for 24 months progression.

Worsening of ILD was observed in $11.7 \%$ (23/196) and 19.9\% (65/326) of LcSSc patients with ILD at baseline, at 12 and 24 months follow-up respectively. In multivariate analysis, variables predicting ILD progression at 24 months were EScSG$\mathrm{Al}>3$ (OR [95 IC]: 3.8 [1.51-9.56], p=0.005), FVC (1.03 [1.01-1.04], $p<0.001)$ and LVEF $(0.91$ [0.85-0.97], $p=0.005)$

Conclusions: It appears that only few LcSSc patients progress for skin fibrosis ; this limits the use of mRSS in this subset and the potential of anti-fibrotic drugs of skin disease. However, a substantial rate of ILD progression was identified as well as relevant predictors. These results support the inclusion of LcSSc patients in SSc-ILD trials evaluating anti-fibrotic drugs. Our predictive models will be helpful to define enriched population in future clinical trials.

Acknowledgements: This work was supported by an unrestricted grant from INVENTIVA

Disclosure of Interest: None declared

DOI: 10.1136/annrheumdis-2018-eular.2626

\section{OP0208 A PROOF-OF-CONCEPT DOUBLE-BLIND RANDOMISED PLACEBO-CONTROLLED TRIAL OF PROBIOTICS IN SYSTEMIC SCLEROSIS ASSOCIATED GASTROINTESTINAL DISEASE}

A.H.L. Low ${ }^{1}$, G.G. Teng ${ }^{2}$, S. Pettersson ${ }^{3}$, P.F. De Sessions ${ }^{4}$, Q. Fan ${ }^{5}$, A. Law ${ }^{1}$ A. Santosa ${ }^{2}$, A. Lim ${ }^{2}$, J. Thumboo ${ }^{1} .{ }^{1}$ Rheumatology and Immunology, Singapore General Hospital, Duke-National University Hospital; ${ }^{2}$ Division of Rheumatology, National University Health System, ${ }^{3}$ Lee Kong Chian School of Medicine, National Technological University, ${ }^{4}$ Genome Institute of Singapore, Agency for Science, Technology and Research; ${ }^{5}$ Centre for Quantitative Medicine, Duke-NUS Medical School, Singapore, Singapore

Background: Hypothesis: Gastrointestinal (Gl) microbiota is a co-founding factor contributing to systemic sclerosis (SSc) and Gl manifestations. Probiotics reduced $\mathrm{Gl}$ symptoms by modulating microbiome composition in an open-label study.

Objectives: To determine whether probiotics result in reduction of Gl symptoms in SSc patients, assessed using the UCLA Gastrointestinal Tract questionnaire (GIT 2.0)

Methods: In this double-blind placebo-controlled trial, 40 subjects with SSc (total GIT0.1) were randomised to receive 60 days of probiotics (900 billion units/day, composite of lactobacilli, bifidobacteria and streptococcus) or placebo, followed by 60 days of probiotics in both groups. Subjects on probiotics or antibiotics 30 days prior were excluded. Enrolled subjects were required to have stable doses of prednisolone, immunosuppression and $\mathrm{Gl}$ medications 30 days prior and during the trial. Between group differences in total GIT change was assessed after 60 days (primary endpoint) and 120 days (secondary endpoint). Stool microbiome composition was analysed using $16 \mathrm{~S}$ next generation sequencing. We performed principle coordinate analysis, alpha diversity and taxonomic level analyses. Twosample t-tests were used to evaluate between-group differences, reported as mean $\pm S D$. An intention-to-treat and last observer carried forward analysis was done. $P$-value $<0.05$ was considered statistically significant.

Results: 40 subjects were randomised to placebo $(n=21)$ or probiotics $(n=19)$. Baseline characteristics are summarised in table 1. At the primary endpoint change in total GIT was not statistically significant between placebo $(-0.14 \pm 0.27)$ and probiotic groups $(-0.13 \pm 0.31 ; p=0.85)$. At the secondary endpoint, there was greater reduction in total GIT in the probiotic $(n=13 ;-0.18 \pm 0.26)$ than the initial placebo group $(n=15 ;-0.05 \pm 0.22)$, though not reaching statistical significance $(p=0.14)$. There was a statistically significant reduction in GIT-reflux subdomain in the probiotic group $(-0.22 \pm 0.16$ vs initial placebo group $0.05 \pm 0.27 ; p=0.0037)$. Subjects on probiotics had greater abundance of lactobacillus, bifidobacterium and streptococcus, and exhibited high alpha diversity, whereas those on placebo had a decreasing trend of alpha diversity. Majority of adverse events were grades land I.
Abstract OP0208 - Table 1 Baseline characteristics of subjects

\begin{tabular}{|c|c|c|}
\hline & Probiotic $(n=19)$ & Placebo $(n=21)$ \\
\hline Fulfill EULAR/ACR 2013 criteria, n (\%) & $19(100)$ & $21(100)$ \\
\hline Mean \pm SD Age, years & $51.4(13.7)$ & $50.7(7.9)$ \\
\hline Females, $\mathrm{n}(\%)$ & $19(100)$ & $16(76.2)$ \\
\hline Disease duration from non- Raynaud's onset, years & $44.5(14.2)$ & $41.3(9.7)$ \\
\hline Diffuse subtype, $n(\%)$ & $3(15.8)$ & $6(28.6)$ \\
\hline Anti-Centromere positive, $\mathrm{n}(\%)$ & $7(36.8)$ & $2(9.5)$ \\
\hline Anti-Topo-I positive, $n(\%)$ & $2(10.5)$ & $10(47.6)$ \\
\hline Mean \pm SD total GIT & $0.42(0.29)$ & $0.41(0.27)$ \\
\hline Mean \pm SD GIT Reflux & $0.45(0.44)$ & $0.39(0.39)$ \\
\hline Mean + SD GIT Distension & $1.13(0.70)$ & $0.93(0.74)$ \\
\hline Mean + SD GIT Diarrhoea & $0.26(0.44)$ & $0.36(0.60)$ \\
\hline Mean \pm SD GIT Fecal soilage & $0.26(0.71)$ & $0(0)$ \\
\hline Mean + SD Body Mass Index & $23.6(2.8)$ & $22.9(3.0)$ \\
\hline Hydrogen breath test positive, $n(\%)$ & $2(10.5)$ & 3 (14.3) \\
\hline Prednisolone, $n(\%)$ & $6(31.6)$ & $8(38.1)$ \\
\hline GI medications & $13(68.4)$ & $18(85.7)$ \\
\hline Gl: Proton-pump inhibitor & $11(57.9)$ & $17(81.0)$ \\
\hline GI: Promotility agent & $7(36.8)$ & 7 (33.3) \\
\hline Immunosuppressive medications & $10(52.6)$ & $13(61.9)$ \\
\hline
\end{tabular}

Conclusions: This trial demonstrated safety of probiotics in SSc. The primary outcome at 60 days was not achieved. A prolonged course of probiotics (120 days) resulted in greater improvement of GI reflux. There was a possible positive association between reduced GI stress (evidenced by greater alpha diversity of the GI microbiota) and lower GIT scores in the probiotic group. This study provides justification for a larger definitive trial of probiotics in SSc.

REFERENCE:

[1] Frech TM, Khanna D, Maranian P, et al. Probiotics for the treatment of systemic sclerosis-associated gastrointestinal bloating/distention. Clin Exp Rheumatol 2011;29(2 Suppl 65):S22-25

Disclosure of Interest: None declared

DOI: 10.1136/annrheumdis-2018-eular.4623

THURSDAY, 14 JUNE 2018

\section{Patient involvement in research: The future of collaborative research. Lessons from the field of rheumatology and beyond}

\section{OP0209-PARE THE PATIENT VOICE IN ARTHRITIS RESEARCH: A COLLABORATIVE APPROACH TO EMBEDDING PPI INTO RESEARCH STRATEGY}

N. Nestor, on behalf of The Patient Voice in Arthritis Research Patient Insight Partners, A.G. Wilson, E.R. Dorris. Centre for Arthritis Research, University College Dublin, Dublin, Ireland

Background: Public and Patient Involvement (PPI) encompasses a variety of ways researchers engage with people for whom their research holds relevance. Active and formal PPI can result in increased patient support for research and improved likelihood of patient involvement in the case of clinical research, including improved relevance to patient quality of life. As a research centre, we decided to develop our own PPI initiative. In order to develop a meaningful and productive partnership, we have developed this initiative from conception with our patient insight partners.

Objectives: The overall objective is to improve our research quality, relevance and outcomes. We aim to ensure that the real-life experiences of people living with arthritis are considered in the decision making processes around arthritis research.

Methods: A community approach to recruitment of patient insight partners was used. Social media, advocacy charities, and local community events were the pre dominant source of recruitment. Three initiatives were proposed: steering committee, patient insight panels, and a patient educator programme. A discussion forum between patients and researchers was used to determine the feasibility, interest and accessibility of proposed initiatives. An independent facilitator was commis sioned to prepare an unbiased report of the discussion forum, upon which the PP strategy was developed.

Results: Patient support for the PPI initiative was overwhelmingly positive. A number of potential barriers to participation were identified.

1) Steering committee:

Risk of tokenism and the potential intimidation of a structure that was too formal Mechanisms to overcome included multiple patient representatives, detailed terms of reference and a supportive environment with a rotating chair. Our patient insight partners proposed a three-tier structure: a patient focus group that 
nominates the steering committee members and a plenary meeting to be kept informed about research they are involved in.

2) Insight Panels:

Access to technology was viewed as the major barrier to remote involvement. Mechanisms to overcome included multiple modes of communication: online, tele phone, postal communication. Providing the opportunity for face-to-face or speak ing directly with a researcher in an informal setting was seen as crucial in building interpersonal relationships and sustaining involvement.

3) Patient Educator:

Extremely well received. Barriers to participation revolved largely around travel and physical accessibility. This can be overcome with in-house resources.

Our research strategy is being revised with the PPI strategy as a central tenet. We are adopting the new steering committee under the three-tier format and yearly research meeting with plenary session. We have a PPI newsletter, the editorial board for which is made up of researchers and patient insight partners. A patient educator module is under review for incorporation into a new PhD programme. We have expanded our research into multidisciplinary areas, with new sociology researchers and psychology collaborators.

Conclusions: The development a true patient partnership in our group has fundamentally changed the scope and remit of our research, allowing us to expand our biomedical and clinical research into a more holistic model.

Disclosure of Interest: None declared

DOI: 10.1136/annrheumdis-2018-eular.6815

\section{OP0210-PARE DUTCH JUVENILE IDIOPATHIC ARTHRITIS PATIENTS, CARERS AND CLINICIANS CREATING A RESEARCH AGENDA TOGETHER FOLLOWING THE JAMES LIND ALLIANCE METHOD}

C.G Schoemaker ${ }^{1,2}$, W. Armbrust ${ }^{3,4}$, J.F. Swart ${ }^{2,4}$, E. Versluis $^{1}$, W. Olsder ${ }^{5}$, N M. Wulffraat ${ }^{2} .{ }^{1}$ Dutch JIA patient organisation, member of ENCA, Rijen;

${ }^{2}$ Department of Pediatric Rheumatology, University Medical Center Utrecht, Utrecht, ${ }^{3}$ Department of Pediatric Rheumatology, University Medical Center Groningen, Groningen; ${ }^{4}$ Netherlands Association for Pediatric Rheumatology, Utrecht, ${ }^{5}$ Youth-R-Well.com, Young Patient Organization, Lisse, Netherlands

Background: Biomedical research should support patients, carers and clinicians to take important decisions in the consulting room and eventually to improve the lives of patients. 'Thus far the end-users of Juvenile Idiopathic Arthritis (JIA) research have not been involved in the prioritisation of future research. The JIA research community clearly sees the unmet need and has repeatedly expressed the wish to do something about this. As Parsons et al. have put it: 'Understanding young people's research priorities is important to develop research that is in tune with their needs'. 'P Putting this into practice starts with a search for relevant issues working together with the end users of scientific knowledge on JIA - patients, carers and clinicians - and to prioritise research questions that can really make a difference.

Objectives: In 2018 Dutch organisations of patients, carers and clinicians will collaboratively develop a research agenda for JIA, following the James Lind Alliance methodology. ${ }^{3,4}$ An established research agenda, created by patients, carers and clinicians, will inform researchers and research funders about what the most important, relevant research questions for JIA are.

Methods: The James Lind Alliance (JLA) methodology enables us to do a sys tematic search for unanswered questions that are relevant to patients, carers and clinicians. 'In a 'Priority Setting Partnership' (PSP), we will gradually establish a top 10 list of the most important unanswered research questions for JIA. In this process the input from patients and their carers will be given the same weight as that from clinicians.

The Dutch JIA PSP will be led by a steering group. This steering group coordinates the PSP and organises the activities. It will include representatives of patients (for JIA: young and adult JIA-patients), carers (for JIA: parents and spouses) and clinicians (for JIA: paediatric rheumatologists, physiotherapists, nurses, psychologists, social workers, ophthalmologist, etc.).

Results: The Dutch JIA organisations support the agenda; also financially. Following the JLA method it will take approximately twelve to eighteen months to for mulate a research agenda, so te research agenda for JIA will be published in 2019.
Conclusions: A research agenda that will be jointly inspired by patients. carers and clinicians can really make a difference for decision-making in the consulting room and for the lives of JIA-patients.

\section{REFERENCES}

[1] Macleod MR, Michie S, Roberts I, et al. Biomedical research: increasing value, reducing waste. Lancet 2014;383:101-4. doi:10.1016/S0140-6736 (13)62329-6

[2] Parsons S, Thomson W, Cresswell K, Starling B, McDonagh JE. Barbara Ansell, National Network for Adolescent Rheumatology. What do young people with rheumatic disease believe to be important to research about their condition? A UK-wide study. Pediatr Rheumatol Online J 2017;15:53.

[3] Schoemaker CG, Prakken AB, Furth EF. Patients and physicians creating a research agenda together: the method of the British James Lind Alliance [Article in Dutch]. Ned Tijdschr Geneeskd 2017;161(0):D1764.

[4] JLA Guidebook. www.jla.nihr.ac.uk/jla-guidebook [Accessed 24 Jan 2018].

Disclosure of Interest: None declared

DOI: 10.1136/annrheumdis-2018-eular.2756

\section{THURSDAY, 14 JUNE 2018}

\section{Can we improve the care of gout?}

\section{OP0211 ULTRASOUND SHOWS RAPID REDUCTION OF URIC ACID LOAD DURING A TREAT-TO-TARGET APPROACH IN GOUT PATIENTS: RESULTS FROM A LONGITUDINAL STUDY (NOR-GOUT)}

${ }_{\text {H.B. Hammer }}{ }^{1}$, L. Karoliussen ${ }^{1}$, L. Terslev ${ }^{2}$, E.A. Haavardsholm ${ }^{1,3}$, T.K. Kvien ${ }^{1}$, T. Uhlig ${ }^{1}{ }^{1}$ Dept Of Rheumatology, Diakonhjemmet hosptial, Oslo, Norway, ${ }^{2}$ Centre for Rheumatology and Spinal Diseases, Copenhagen University Hospital at Glostrup, Copenhagen, Denmark; ${ }^{3}$ University of Oslo, Oslo, Norway

Background: Ultrasound (US) has received an increasing attention in detecting uric monosodium urate (MSU) deposits, and is included as a domain in the ACR/ EULAR classification criteria for gout. The OMERACT US group has developed definitions for US elementary lesions in gout including the double contour sign (DC) (deposits of crystals on the surface of cartilage), tophus (larger hypo-echoic aggregation of crystals, usually well delineated), aggregates (small hyper-echoic deposits) and erosions. MSU deposits may be found in many different regions with some predilection sites, but only a few small studies have explored the decrease of deposits during treatment.

Objectives: To explore by US the longitudinal resolution of MSU deposits during a treat-to-target approach with urate lowering therapy (ULT) in patients with gout.

Methods: In a prospective observational study, patients with crystal-proven gout were included if they presented after a recent gout flare and had increased serum urate levels (>360 $\mu \mathrm{mol} / \mathrm{L} />6 \mathrm{mg} / \mathrm{dl}$ ). In a treat-to-target approach using ULT and increasing drug doses with monthly follow-up until treatment target was met $(<360$ $\mu \mathrm{mol} / \mathrm{L}$, or $<300 \mu \mathrm{mol} / \mathrm{L}$ if clinical tophi). An extensive US assessment was performed (GE E9 machine, grey scale $15 \mathrm{MHz}$ ) at baseline and after 3, 6 and 12 months to detect MSU deposits, using the OMERACT definitions for DC, toph and aggregates with bilateral assessment of radiocarpal joints, MCP 2, insertion of triceps and quadriceps, proximal and distal patellar tendon, the Achilles tendon and cartilage of distal femur (maximal flexed knee) and the talar cartilage of tibiotalar joint and MTP 1 joint. The degree of elementary lesions was semi-quantitatively scored $0-3$ ( 0 =none, $1=$ possible, $2=$ certain, $3=$ major deposits). Sum scores of DC, tophi and aggregates, as well as total sum score of all lesions, were calcu lated for each visit. Changes from baseline were explored by paired samples Ttest and response by Standardised Response Mean (SRM).

Results: 161 patients were included at baseline (93.3\% men, mean (SD) age 57.0 (14.1) years, disease duration 8.0 (7.7) years). The mean (SD) serum urate level decreased from 487 (82) $\mu \mathrm{mol} / \mathrm{L}$ at baseline to $312(52) \mu \mathrm{mol} / \mathrm{L}$ at 12 months, with $72 \%$ reaching the target at 3 months, and $84 \%$ at 12 months. Sum scores of deposits decreased over 12 months (table 1 , with ${ }^{*}=p<0.05,{ }^{* *}=p<0.001$ ), and the numeric decrease was largest for DC (figure 1). SRM from baseline to 3, 6 and 12 months was $0.73,1.02$ and 1.26 for DC, $0.06,0.57$ and 0.91 for tohpi and 0.20 0.51 and 0.66 for aggregates. 